\title{
Evaluación de las experiencias de tipo psicótico en adolescentes españoles: Baremación del Prodromal Questionnaire-Brief (PQ-B)
}

\author{
Eduardo Fonseca-Pedrero ${ }^{1,2}$, Alicia Pérez-Albéniz ${ }^{1}$, Javier Ortuño-Sierra ${ }^{1}$ y Félix Inchausti ${ }^{3}$ \\ ${ }^{1}$ Universidad de La Rioja, Logroño, España \\ ${ }^{2}$ Universidad de Oviedo, Oviedo, España \\ ${ }^{3}$ Servicio Riojano de Salud, Logroño, España
}

\begin{abstract}
Assessment of psychotic-like experiences in Spanish adolescents: Normative data of the Prodromal Questionnaire-Brief (PQ-B)
\end{abstract}

\begin{abstract}
The main objective of this work was to establish the normative data of the Prodromal Questionnaire-Brief (PQ-B) in a representative sample of Spanish adolescents. A total of 3,441 adolescents ( $M=15.91$ years, $S D=1.33$ years) were selected by sampling stratified by clusters. The results showed that the point prevalence of psychotic-like experiences varied between $12 \%$ and $48 \%$. An $82.9 \%$ of the sample reported at least one attenuated psychotic-like experience. Percentile norms were made for the total score and according to gender. The PQ-B is a brief, simple, useful measuring instrument with adequate psychometric properties for the assessment of subclinical psychosis phenotype in the Spanish adolescent population. It is transcendental that the psychologists have adequate tools for a reliable and valid assessment of this phenomenon in order to make informed decisions and optimize educational and socio-health resources.
\end{abstract}

Keywords: Psychosis; psychotic experiences; adolescents; youth; validation; norms.

Resumen: El principal objetivo de este trabajo fue baremar el Cuestionario de Pródromos-Breve (Prodromal QuestionnaireBrief, PQ-B) a partir de una muestra representativa de adolescentes españoles. Un total de 3441 adolescentes $(M=15.91$ años, $D T=1.33$ años) fueron seleccionados mediante muestreo estratificado por conglomerados. Los resultados mostraron que la prevalencia puntual de experiencias psicóticas osciló entre el $12 \%$ y el $48 \%$. Un $82.9 \%$ de la muestra refirió al menos una experiencia psicótica atenuada. Los baremos tipo percentiles se confeccionaron para la puntuación total y en función del género. El PQ-B es un instrumento de medida breve, sencillo, útil y con adecuadas propiedades psicométricas para la valoración de las experiencias de tipo psicótico en población adolescente española. Es transcendental que el profesional de la psicología disponga de adecuadas herramientas para la evaluación fiable y válida de este fenómeno de cara a tomar decisiones fundamentadas y optimizar los recursos educativos y socio-sanitarios.

Palabras clave: Psicosis; experiencias de tipo psicótico; adolescentes; jóvenes; validación; baremación.

Recibido: 27 de febrero 2020; aceptado: 06 de julio 2020

Correspondencia: Dr. Eduardo Fonseca Pedrero, Departamento de Ciencias de la Educación, Universidad de La Rioja, C/ Luis de Ulloa, 2 (Edificio Vives), 26004 Logroño, España. Correo-e: eduardo.fonseca@unirioja.es

Agradecimientos: Esta investigación ha sido financiada por la Convocatoria 2015 de Ayudas Fundación BBVA a Investigadores y Creadores Culturales, por las Ayudas Fundación BBVA a Equipos de Investigación Científica 2017, el Instituto de Estudios Riojanos (IER) y cofinanciado con fondos FEDER en el PO FEDER de La Rioja 20142020 (SRS 6FRSABC026).

\section{Introducción}

Las experiencias psicóticas forman parte de la diversidad humana. Por ejemplo, oír voces, como cualquier otro fenómeno psicológico, surge como respuesta a un contexto biográfico y social determinado que es vivenciado por una persona concreta (Cooke, 2014; Fonseca-Pedrero, 2019). El modelo dimensional de psicosis considera que el fenotipo psicótico se distribuye a lo lar- 
go de un continuo de gravedad y, por lo tanto, que las experiencias psicóticas pueden estar presentes en la población general. A lo largo de este continuum psico(pato)lógico es posible encontrar expresiones no clínicas (p.ej., rasgos esquizotípicos, experiencias psicóticas atenuadas), subclínicas (p.ej., síntomas psicóticos atenuados, estados mentales de alto riesgo) y clínicas (p.ej., trastornos del espectro psicótico) (Kwapil y Barrantes-Vidal, 2015; Linscott y van Os, 2013).

Al conjunto de fenómenos pseudo-psicóticos que no se asocian necesariamente con psicopatología (alteración médica o de otro tipo) ni con malestar, discapacidad o búsqueda de tratamiento, que se pueden presentar en la población general y que se extienden más allá de las fronteras clínicas establecidas por los manuales diagnósticos, se les conoce como experiencias psicóticas atenuadas (naturaleza estado) o rasgos esquizotípicos (naturaleza rasgo) (Fonseca-Pedrero et al., 2021). Este acercamiento, que intenta analizar y comprender las experiencias psicóticas atenuadas en muestras no clínicas, se ha denominado «fenotipo psicótico extendido»-o ampliado- (van Os y Linscott, 2012). Dichas experiencias son de naturaleza multidimensional y se agrupan, básicamente, en las facetas Positiva (p.ej., experiencias perceptivas inusuales), Negativa (p.ej., anhedonia) y de Desorganización Cognitiva (p.ej., pensamiento desorganizado) (p.ej., Fonseca-Pedrero et al., 2018; Stefanis et al., 2002).

La prevalencia de las experiencias psicóticas en muestras de la población general ha sido ampliamente analizada (McGrath et al., 2015; Nuevo et al., 2012). En meta-análisis previos se ha encontrado una incidencia media anual del $2.5 \%$ y una prevalencia media anual del $7.2 \%$ (Linscott y van Os, 2013). Por su parte, la prevalencia media en niños de 9 a 12 años es del 17\%, mientras que en adolescentes de 13 a 18 años es del 7.5\% (Kelleher et al., 2012). Dichas experiencias suelen tener un carácter transitorio durante la adolescencia, si bien en un pequeño porcentaje de casos pueden volverse anormalmente persistentes y evolucionar hacia discapacidad y necesidad de tratamiento (Cougnard et al., 2007).

El estudio de las experiencias psicóticas atenuadas y los síntomas psicóticos subclínicos se fundamenta, básicamente, en la posibilidad de comprender mecanismos etiológicos subyacentes a los trastornos del espectro psicótico (Fonseca-Pedrero y Inchausti, 2018). Conocer potenciales factores de riesgo y de protección, así como marcadores etiológicos, puede ayudar a establecer y optimizar estrategias de prevención, básicamente detección precoz e intervención temprana en psicosis ( Fusar-Poli et al., 2020; Fusar-Poli et al., 2017). Estudios longitudinales previos han encontrado que aquellos indi- viduos de la población general que informan de experiencias psicóticas o rasgos esquizotípicos tienen un mayor riesgo de desarrollar un trastorno del espectro psicótico en el futuro (Debbané et al., 2015; Kaymaz et al., 2012; Zammit et al., 2013), así como diversos problemas de salud mental (p.ej., depresión) (Fisher et al., 2013); no obstante, los niveles de especificidad encontrados en la predicción de trastornos psicóticos son bajos.

Por ello, estas experiencias no se pueden considerar una condición necesaria ni suficiente para el desarrollo posterior de un trastorno psicótico (u otro trastorno mental), siendo necesario examinar su asociación con otros factores (p.ej., trauma, consumo de cannabis) (Bak et al., 2005; Fonseca-Pedrero, 2018b; Fusar-Poli et al., 2017; Kelleher et al., 2013; Linscott y van Os, 2013). Además, estudios previos han demostrado que dichas experiencias se asocian con los mismos factores de riesgo genético, sociodemográficos y ambientales encontrados en personas con diagnóstico de psicosis. Por ejemplo, entre otros, se han señalado como factores de riesgo la desregulación afectiva, las experiencias de trauma, el uso y consumo de cannabis y alcohol o la historia familiar de trastorno mental (p.ej., Dolphin et al., 2015; Fonseca-Pedrero et al., 2017; Fonseca-Pedrero et al., 2021; Linscott y van Os, 2013; Strauss et al., 2018). En general, los trabajos publicados hasta la fecha sugieren la validez y utilidad de las experiencias psicóticas subclínicas en la comprensión y prevención de la psicosis.

Se han formulado multitud de hipótesis para explicar el posible origen del síndrome de psicosis. Aquí no se pretende realizar una revisión exhaustiva, por lo que remitimos al lector a excelentes trabajos previos (Fonseca-Pedrero, 2019; Howes y Murray, 2014; Kahn et al., 2015; Lemos-Giráldez et al., 2015; Millan et al., 2016; Pérez-Álvarez, 2012; Radua et al., 2018). No cabe duda de que analizar y comprender la conducta humana, en este caso la psicosis, es un asunto harto complejo. Posiblemente, la respuesta esté en la intrincada interacción dinámica que se establece entre los factores biológicos, psicológicos y sociales, experimentados (vivenciados) por una persona concreta con una biografía particular y unas circunstancias determinadas. Es sabido que la conducta humana, por su enorme complejidad y diversidad, se ajusta mal a lo lineal, a lo estático y a lo unicausal, siendo necesaria una perspectiva individual, propositiva, multidimensional, multifactorial, adaptativa, dinámica y contextual (p.ej., Fonseca-Pedrero, 2019; Pérez-Álvarez, 2018). Por ello, los modelos más actuales en este área de estudio buscan poner nuevamente de relieve la necesidad de considerar a la persona, que no al cerebro, desde una perspectiva fenomenológica, que no biológica (Pé- 
rez-Álvarez et al., 2010; Pérez-Álvarez y García-Montes, 2018). Más que estudiar el órgano cerebral, habría que estudiar el organismo; más que analizar al enfermo o paciente se debería considerar a la persona; más que analizar una posible causa latente subyacente habría que entender la psicosis como un sistema complejo dinámico (Borsboom, 2017; Fonseca-Pedrero, 2018a).

La evaluación fiable y válida de las experiencias psicóticas en jóvenes es esencial. Sin una correcta evaluación y posterior diagnóstico, difícilmente se podrá realizar una intervención acertada (Muñiz y Fonseca-Pedero, 2019) y, por lo tanto, dar una atención de calidad a las personas. Obviamente, una correcta evaluación reclama no solo que el profesional de la psicología tenga una buena formación, sino que es necesario que disponga, además, de instrumentos con adecuadas propiedades psicométricas, que se hayan construido y adaptado siguiendo los estándares internacionales y estén validados y baremados para un uso, población y contexto específico (Hernández et al., 2016; Muñiz y Fonseca-Pedrero, 2019). Sea como fuere, no deja de ser curioso que, considerando la relevancia clínica y científica de la psicosis y el rol del psicólogo en su evaluación y tratamiento, la utilización de test para la evaluación del fenotipo psicótico es inexistente entre los psicólogos españoles (Muñiz et al., 2020).

En los últimos años se han desarrollado y validado una amplia variedad de instrumentos para la evaluación de las diferentes expresiones del fenotipo psicótico a nivel poblacional, subclínico y clínico (p.ej., Fonseca-Pedrero, 2018b; Fernández-León et al., 2019). Al acercamiento metodológico que trata de estudiar las experiencias psicóticas en muestras de la población general se le conoce como «paradigma de alto riesgo psicométrico», mientras que al estudio de personas con síntomas subclínicos (estado mental de alto riesgo de psicosis) se le conoce como «paradigma de alto riesgo clínico». Estos métodos de investigación son considerados estrategias fiables y válidas para la detección e identificación precoz del riesgo a la psicosis (p.ej., Fusar-Poli et al., 2014), siendo también útiles de cara a la posible implementación posterior de tratamientos profilácticos (p.ej., Fusar-Poli et al., 2017, 2020). Las propiedades psicométricas de estas herramientas de evaluación han sido revisadas extensamente en trabajos previos (p.ej., Fonseca-Pedrero et al., 2016; Kline y Schiffman, 2014; Savill et al., 2018).

Dentro del paradigma de alto riesgo clínico se pueden encontrar instrumentos de tipo autoinforme como el Youth Psychosis At-Risk Questionnaire (Y-PARQ) (Ord et al., 2004) y el Prodromal Questionnaire (PQ) con sus versiones extendida (Loewy et al., 2005) y breve (PQ-B)
(Loewy et al., 2011). Estos cuestionarios se han mostrado adecuados para la evaluación de los síntomas psicóticos atenuados en jóvenes y permiten obtener evidencias sobre la presencia, frecuencia y gravedad de dichas experiencias y síntomas (Kline y Schiffman, 2014; Savill et al., 2018). Cierto es que este tipo de instrumentos se han desarrollado inicialmente en el campo del alto riesgo clínico, si bien dentro del modelo dimensional del fenotipo psicótico también se han utilizado en muestras de la población general.

En concreto, el cuestionario de Pródromos (PQ) (de psicosis) y su versión abreviada (PQ-B) destacan como unos de los instrumentos más utilizados en este campo. El PQ es un autoinforme compuesto por 92 ítems que valoran síntomas prodrómicos de psicosis. Los mismos autores del PQ desarrollaron el PQ-B con la finalidad de tener una herramienta breve que permitiera una evaluación rápida, fiable y precisa del fenotipo psicótico subclínico. La versión abreviada de 21 ítems valora, esencialmente, la dimensión positiva del fenotipo de psicosis, ya que es la base de los síndromes prodrómicos (p.ej., estados mentales de alto riesgo). Estudios previos han mostrado que el PQ-B también es una herramienta interesante en la detección de estados mentales de alto riesgo (sensibilidad y especificidad) en jóvenes (Kline y Schiffman, 2014), así como en adolescentes y adultos de la población general (Cicero et al., 2019; Fonseca-Pedrero et al., 2018). Esta versión corta resulta sumamente interesante para utilizar como instrumento de cribado en contextos educativos, sociales y/o clínicos en una primera evaluación clínico-psicopatológica. Asimismo, puede ser de utilidad en investigaciones donde los recursos temporales y/o materiales son limitados, pero en las que se quiere recabar información sobre esas experiencias.

El PQ-B se encuentra validado en adolescentes españoles (Fonseca-Pedrero et al., 2016). Los resultados de este estudio mostraron que las puntuaciones del PQ-B presentan adecuadas propiedades psicométricas. Los análisis factoriales confirmatorios arrojaron una estructura esencialmente unidimensional. Dicha estructura se mostró invariante en función del género. La consistencia interna de las puntuaciones del PQ-B, estimada con el Omega de McDonald, fue de 0.92. La función de información del test mostró que se medía con mayor precisión los niveles superiores del rasgo latente. Las puntuaciones del PQ-B se asociaron positivamente con psicopatología, problemas emocionales y comportamentales, conducta suicida y síntomas de depresión (Fonseca-Pedero et al., 2020; Fonseca-Pedrero et al., 2016; Fonseca-Pedrero et al., 2018). No obstante, hasta el momento, en España existen pocas herramientas de medición, como el PQ-B, que permitan la evaluación de las 
experiencias psicóticas atenuadas en adolescentes y que se encuentran debidamente baremadas.

Dentro de este contexto de investigación, el principal objetivo del presente trabajo fue realizar la baremación del PQ-B en una muestra representativa de adolescentes españoles.

\section{Método}

\section{Participantes}

Con la finalidad de garantizar la representatividad de la muestra, se realizaron, durante los años 2016 y 2019, dos muestreos aleatorios estratificados por conglomerados, a nivel de aula, en una población aproximada de quince mil estudiantes pertenecientes a la Comunidad Autónoma de La Rioja. Los estratos se crearon en función del centro escolar (público/privado-concertado) y la etapa escolar (ESO, Bachillerato y Formación Profesional), donde la probabilidad de extracción del aula del centro escolar venía determinada en función del número de alumnos.

Participaron un total de $N=3.441$ adolescentes, $53.4 \%$ mujeres $(n=1.843)$, con edades comprendidas entre los 14 y 18 años $(M=15.91$ años, $D T=1.33$ años). La primera muestra estuvo formada por 1.664 participantes $(M=16.12$ años; $D T=1.36$ años $)$, siendo el 53\% mujeres y la segunda por $1.777(M=15.70$ años; $D T=$ 1.26 años), siendo el $53.7 \%$ mujeres. La distribución por grupos de edad fue: 14 años $(n=562), 15$ años $(n=898)$, 16 años $(n=818) ; 17$ años $(n=699) ;$ y 18 años $(n=$ 464). La distribución de la nacionalidad de los participantes fue la siguiente: $89.4 \%$ española, $2.5 \%$ rumana, 1.9\% latinoamericana (Bolivia, Argentina, Colombia y Ecuador), $1.4 \%$ marroquí, $0.8 \%$ pakistaní, $0.3 \%$ portuguesa y $3.8 \%$ de otras nacionalidades.

Para garantizar la validez del proceso de repuesta, se eliminaron aquellos participantes que presentaban un patrón de respuesta azaroso o pseudoazaroso, utilizando la Escala Oviedo de Infrecuencia de Respuesta (Fonseca-Pedrero et al., 2009).

\section{Instrumentos}

Cuestionario de Pródromos-Breve (Prodromal Questionnaire-Brief; PQ-B; Loewy et al., 2011). El PQ-B es un autoinforme compuesto por 21 ítems que valoran síntomas prodrómicos de psicosis de tipo positivo. Los ítems se encuentran formulados en formato dicotómico verdadero/falso. Si el participante responde afirmativamente al ítem, tiene que indicar el grado de preocupación o malestar que le provoca dicha experiencia en una escala tipo Likert de cinco opciones de respuesta (de $1=$ totalmente en desacuerdo a $5=$ totalmente de acuerdo). Una mayor puntuación indica mayor número de experiencias psicóticas, así como mayor gravedad. La consistencia interna en este estudio, estimada con el coeficiente omega, fue 0.91. El PQ-B, adaptado a población española, se presenta en el Anexo 1.

Escala Oviedo de Infrecuencia de Respuesta (INFOV; Fonseca-Pedrero et al., 2009). La INF-OV se ha desarrollado para detectar a aquellos participantes que han respondido de forma azarosa, pseudoazarosa o deshonesta a los instrumentos de medida administrados. La INF-OV es un instrumento de medida tipo autoinforme compuesto por 12 ítems en formato tipo Likert de cinco categorías en función del grado de adherencia $(1=\mathrm{com}$ pletamente en desacuerdo; 5 = completamente de acuerdo). Una vez dicotomizados los ítems, los alumnos que responden más de dos ítems de la INF-OV de forma incorrecta son eliminados del estudio.

\section{Procedimiento}

Las dos investigaciones fueron aprobadas por la Dirección General de Educación del Gobierno de La Rioja y el Comité Ético de Investigación Clínica de La Rioja (CEICLAR). Con la finalidad de estandarizar el proceso de administración, a todos los investigadores se les entregó un protocolo y unas normas que debían llevar a cabo antes, durante y después de la administración de los instrumentos de medida. La administración de los cuestionarios se realizó por ordenador y de forma colectiva en grupos de entre 10 y 30 participantes.

En todo momento se informó al estudiantado de la confidencialidad de las respuestas, así como del carácter voluntario de la participación y no se dio gratificación alguna por la colaboración en el estudio. Se solicitó un consentimiento por parte de las familias que autorizase la participación de cada adolescente en la investigación.

\section{Análisis estadístico}

Primero, se calcularon los estadísticos descriptivos (media, desviación típica, asimetría y curtosis) para los ítems del PQ-B y se analizó la proporción de respuestas afirmativas en cada ítem del PQ-B. En segundo lugar, se analizó si existían diferencias estadísticamente significativas en las puntaciones totales del PQ-B en función del género y la edad. Para ello se realizó un análisis multivariado de la varianza (MANOVA), donde el género y la edad fueron consideradas variables independientes. El $\eta^{2}$ parcial se utilizó como indicador del tamaño del efecto. En tercer lugar, se elaboraron baremos tipo percentil 
para la puntuación total del PQ-B tanto para la muestra total como en función del género. También calcularon los estadísticos descriptivos para las puntuaciones totales del PQ-B. Se utilizó el SPSS 22.0 para realizar el análisis de datos.

\section{Resultados}

\section{Estadísticos descriptivos}

Los resultados de los estadísticos descriptivos de los ítems del PQ-B y la prevalencia de experiencias psicóticas atenuadas autoinformadas se recogen en la Tabla 1. Como se puede observar, la prevalencia puntual osciló entre un $12 \%$ (ítem 3 ) y $48 \%$ (ítems 8 y 18 ). Un $82.9 \%$ de la muestra refirió al menos una experiencia psicótica atenuada.

El MANOVA mostró el efecto principal del género $\left(\lambda=0.987, F_{(2,3430)}=23.299 ; p<0.001 ; \eta^{2}\right.$ parcial $=$ $0.013)$ y la edad $\left(\lambda=0.995, \mathrm{~F}_{(8,6860)}=2.222 ; p=0.023\right.$; $\eta^{2}$ parcial $=0.003$ ).

En función del género, los contrastes de los ANOVAS univariados indicaron que las mujeres presentaron puntuaciones medias más altas que los hombres en la puntuación total del PQ-B de frecuencia $\left(F_{(1,3431)}=\right.$ 45.984; $p<0.001 ; \eta^{2}$ parcial $=0.013 ; M_{\text {hombre }}=5.06$. $\left.D T_{\text {hombre }}=4.22 ; M_{\text {mujer }}=6.14, D T_{\text {mujer }}=4.38\right)$ y en malestar asociado $\left(F_{(1.3431)}=8.984 ; p=0.003 ; \eta^{2}\right.$ parcial $=0.003$; $M_{\text {hombre }}=6.99, D T_{\text {hombre }}=11-79 ; M_{\text {mujer }}=8.27, D T_{\text {mujer }}=$ 12.85).

En función de la edad, los resultados de los contrastes ANOVAS univariados revelaron un valor $p=0.064$ $\left(F_{(4.3431)}=2.224 ; p=0.064 ; \eta^{2}\right.$ parcial $\left.=0.003\right)$ para la puntuación total de frecuencia del $\mathrm{PQ}-\mathrm{B}$, mientras que para la puntuación total de malestar fue $p=0.008\left(F_{(4.3431)}\right.$ $=3.483 ; p=0.008 ; \eta^{2}$ parcial $\left.=0.004\right)$. En las comparaciones post hoc de la puntuación total de frecuencia del PQ-B no se encontraron diferentes destacables entre ningún par de grupos de edad. En la puntuación total de malestar del PQ-B únicamente se encontraron diferencias destacables entre los grupos de edad de 14 y 15 años, presentando el grupo de participantes de 14 años puntaciones medias superiores respecto al de 15 años.

Los resultados del MANOVA encontrados para la interacción género-edad fueron: $\lambda=0.996, F_{(8.6860)}=1.880$; $p=0.059 ; \eta^{2}$ parcial $=0.002$.

Tabla 1. Estadísticos descriptivos y prevalencia (\%) de experiencias psicóticas atenuadas en una muestra de adolescentes utilizando el Prodromal Questionnaire-Brief

\begin{tabular}{|c|c|c|c|c|c|c|c|c|}
\hline \multirow[b]{2}{*}{ Ítem } & \multicolumn{4}{|c|}{ Muestra total } & \multicolumn{2}{|c|}{ Hombres } & \multicolumn{2}{|c|}{ Mujeres } \\
\hline & $M$ & $D T$ & Asimetría & Curtosis & $M$ & $D T$ & $M$ & $D T$ \\
\hline 1 & 0.16 & 0.37 & 1.83 & 1.35 & 0.15 & 0.36 & 0.17 & 0.38 \\
\hline 2 & 0.45 & 0.50 & 0.20 & -1.96 & 0.44 & 0.49 & 0.46 & 0.50 \\
\hline 3 & 0.12 & 0.33 & 2.30 & 3.30 & 0.12 & 0.33 & 0.12 & 0.33 \\
\hline 4 & 0.17 & 0.38 & 1.76 & 1.11 & 0.14 & 0.35 & 0.19 & 0.40 \\
\hline 5 & 0.23 & 0.42 & 1.25 & -0.43 & 0.19 & 0.39 & 0.28 & 0.45 \\
\hline 6 & 0.24 & 0.43 & 1.21 & -0.53 & 0.19 & 0.39 & 0.29 & 0.45 \\
\hline 7 & 0.16 & 0.37 & 1.88 & 1.53 & 0.2 & 0.40 & 0.12 & 0.33 \\
\hline 8 & 0.48 & 0.50 & 0.10 & -1.99 & 0.39 & 0.49 & 0.55 & 0.50 \\
\hline 9 & 0.18 & 0.39 & 1.65 & 0.72 & 0.17 & 0.37 & 0.19 & 0.40 \\
\hline 10 & 0.28 & 0.45 & 0.99 & -1.02 & 0.25 & 0.43 & 0.31 & 0.46 \\
\hline 11 & 0.32 & 0.47 & 0.77 & -1.41 & 0.27 & 0.44 & 0.36 & 0.48 \\
\hline 12 & 0.40 & 0.49 & 0.40 & -1.84 & 0.33 & 0.47 & 0.47 & 0.50 \\
\hline 13 & 0.13 & 0.34 & 2.17 & 2.72 & 0.11 & 0.30 & 0.16 & 0.36 \\
\hline 14 & 0.41 & 0.49 & 0.38 & -1.86 & 0.36 & 0.48 & 0.45 & 0.50 \\
\hline 15 & 0.19 & 0.39 & 1.58 & 0.50 & 0.17 & 0.38 & 0.20 & 0.40 \\
\hline 16 & 0.25 & 0.44 & 1.13 & -0.72 & 0.23 & 0.42 & 0.27 & 0.45 \\
\hline 17 & 0.21 & 0.41 & 1.44 & 0.06 & 0.19 & 0.39 & 0.22 & 0.42 \\
\hline 18 & 0.48 & 0.50 & 0.08 & -2.00 & 0.39 & 0.48 & 0.56 & 0.50 \\
\hline 19 & 0.21 & 0.41 & 1.42 & 0.03 & 0.23 & 0.42 & 0.19 & 0.39 \\
\hline 20 & 0.13 & 0.34 & 2.21 & 2.90 & 0.14 & 0.34 & 0.12 & 0.33 \\
\hline 21 & 0.43 & 0.50 & 0.27 & -1.93 & 0.41 & 0.49 & 0.45 & 0.50 \\
\hline
\end{tabular}

Nota. El porcentaje de experiencias autoinformadas se obtiene multiplicando la puntuación media del ítem x 100. 


\section{Baremación del $P Q-B$}

Para la elaboración de los baremos del PQ-B se ha tenido en cuenta únicamente las diferencias encontradas en función del género. En función de la edad se decidió no incluir baremos dado que únicamente se hallaron diferencias entre dos grupos edad en la puntación PQ-B de ma-

Tabla 2. Baremos del Prodromal Questionnaire-Brief en la muestra total y en función del género para la puntuación total de frecuencia

\begin{tabular}{|c|c|c|c|c|}
\hline & Total & Hombre & Mujer & \\
\hline Percentil & & & & Percentil \\
\hline 1 & 0 & 0 & 0 & 1 \\
\hline 5 & 0 & 0 & 0 & 5 \\
\hline 10 & 0 & 0 & 1 & 10 \\
\hline 20 & 1 & 1 & 2 & 20 \\
\hline 30 & 3 & 2 & 3 & 30 \\
\hline 40 & 4 & 3 & 4 & 40 \\
\hline 50 & 5 & 4 & 6 & 50 \\
\hline 60 & 6 & 5 & 7 & 60 \\
\hline 70 & 8 & 7 & 8 & 70 \\
\hline 80 & 10 & 9 & 10 & 80 \\
\hline 90 & 12 & 11 & 13 & 90 \\
\hline 95 & 14 & 13 & 14 & 95 \\
\hline 99 & 17 & 16 & 17 & 99 \\
\hline M & 5.64 & 5.07 & 6.14 & \\
\hline$D T$ & 4.34 & 4.23 & 4.39 & \\
\hline Asimetría & 0.66 & 0.76 & 0.58 & \\
\hline ET de asimetría & 0.04 & 0.06 & 0.06 & \\
\hline Curtosis & -0.29 & -0.17 & -0.35 & \\
\hline ET de curtosis & 0.08 & 0.12 & 0.11 & \\
\hline
\end{tabular}

Nota. ET = error típico.

\section{Discusión}

En España no existen muchos instrumentos de medición debidamente validados y baremados que permitan la evaluación de las experiencias psicóticas en adolescentes. Es bien sabido que si el profesional de la psicología dispone de herramientas con adecuadas propieda- lestar y ninguna en la puntación de frecuencia, siendo además el tamaño del efecto cercano a cero. En la Tabla 2, se presenta la baremación tipo percentil para la puntación total en frecuencia del PQ-B en la muestra total y en función del género. En la Tabla 3, se presenta la baremación en percentiles para la puntación total en malestar del PQ-B tanto para la muestra total como en función del género.

Tabla 3. Baremos del Prodromal Questionnaire-Brief en la muestra total y en función del género para la puntuación total en distrés-malestar.

\begin{tabular}{|c|c|c|c|c|}
\hline & Total & Hombre & Mujer & \\
\hline Percentil & & & & Percentil \\
\hline 1 & 0 & 0 & 0 & 1 \\
\hline 5 & 0 & 0 & 0 & 5 \\
\hline 10 & 0 & 0 & 0 & 10 \\
\hline 20 & 0 & 0 & 0 & 20 \\
\hline 30 & 0 & 0 & 0 & 30 \\
\hline 40 & 0 & 0 & 0 & 40 \\
\hline 50 & 0 & 0 & 0 & 50 \\
\hline 60 & 3 & 2 & 4 & 60 \\
\hline 70 & 8 & 7 & 10 & 70 \\
\hline 80 & 15 & 14 & 16 & 80 \\
\hline 90 & 26 & 25 & 27 & 90 \\
\hline 95 & 35 & 31 & 36 & 95 \\
\hline 99 & 52 & 47 & 54 & 99 \\
\hline$M$ & 7.68 & 7.00 & 8.28 & \\
\hline$D T$ & 12.39 & 11.79 & 12.86 & \\
\hline Asimetría & 2.04 & 2.25 & 1.89 & \\
\hline ET de asimetría & 0.04 & 0.06 & 0.06 & \\
\hline Curtosis & 4.53 & 6.12 & 3.49 & \\
\hline ET de curtosis & 0.08 & 0.12 & 0.11 & \\
\hline
\end{tabular}

Nota. ET = error típico. Para una correcta interpretación de los baremos de malestar tiene que tener presente que la puntuación de malestar siempre necesita que se haya respondido de forma afirmativa el ítem de frecuencia. Además, aunque la persona responda afirmativamente a un ítem de frecuencia es posible que indique que no tiene ningún tipo de malestar asociado a esa experiencia.

des psicométricas (p.ej., fiabilidad, evidencias de validez, baremación) se facilitan las labores de comprensión, evaluación, diagnóstico, tratamiento y prevención, entre otros aspectos. También es transcendental que el profesional de la psicología disponga de adecuadas herramientas de medición de cara a una mejor gestión de los recursos educativos y socio-sanitarios. Por consiguiente, 
el objetivo esencial del presente trabajo fue presentar los baremos del PQ-B en una muestra representativa de adolescentes españoles.

Las tasas de experiencias psicóticas autoinformadas en el presente trabajo, así como las diferencias encontradas en función del género, son similares a las informadas en estudios previos (Kelleher et al., 2012). Por ejemplo, Calkins et al. (2014) en una muestra de 7.054 adolescentes norteamericanos encontraron que entre un $3.8 \%$ y un $17.6 \%$ informó de síntomas psicóticos. Estos resultados son semejantes en adolescentes españoles. Por su parte, Fonseca-Pedrero et al. (2011), utilizando el ESQUIZO-Q, encontraron que el $43 \%$ de la muestra informó de algún síntoma relacionado con el pensamiento mágico, la ideación referencial y/o las experiencias delirantes o alucinatorias. El 8.9\% refirió 4 o más experiencias psicóticas atenuadas. En este mismo estudio, se hallaron diferencias estadísticamente significativas en función del género en ideación referencial e ideación paranoide. De estos trabajos previos se puede desprender que este conjunto de experiencias puede caer dentro de un fenómeno del desarrollo «normal» y, en la mayoría de los casos, desaparece con el tiempo sin progresión a un trastorno clínico. Estos hallazgos también respaldan la existencia de una continuidad fenotípica de tipo psicométrico, esto es, que las experiencias psicóticas se pueden dar en la población general, sin estar necesariamente asociadas a psicopatología.

La construcción de baremos del PQ-B, tanto para la muestra total como en función del género, permite una correcta interpretación de las puntuaciones a partir de los valores normativos del grupo de referencia. Asimismo, disponer de baremos construidos a partir de muestras representativas de la población adolescente española es una nueva fuente de evidencia que ayuda a la toma de decisiones. Cabe mencionar que para una correcta interpretación de los baremos del PQ-B en la puntuación de malestar se debe tener presente que dicha puntuación siempre necesita que se haya respondido de forma afirmativa a un ítem de frecuencia. Además, aunque la persona responda afirmativamente a dicho ítem de frecuencia, es posible que indique que no tiene ningún tipo de malestar asociado a esa experiencia. Asimismo, una correcta interpretación de las puntuaciones del PQ-B requiere de ciertas matizaciones que se comentan a continuación.

En primer lugar, no es fácil delimitar la frontera entre la experiencia «no clínica», «subclínica» y «psicopatológica», por lo que se debe ser sumamente cauteloso a la hora de hacer inferencias. Aunque las experiencias psicotiformes o esquizotípicas experimentadas durante la adolescencia pueden ser consideradas como uno de los principales marcadores de riesgo exofenotípicos a los trastornos del espectro psicótico, no necesariamente se tienen que ver como un indicador de alteración psicopatología subyacente. En este sentido, muchas de estas experiencias forman parte del propio proceso madurativo del adolescente, siendo relativamente comunes y de carácter transitorio (p.ej., Fonseca-Pedrero et al., 2021; Kelleher et al., 2012). Además, dependiendo de la etapa del desarrollo en la que se refieran (p.ej., niñez, adolescencia temprana o adolescencia tardía), pueden tener un diferente significado psico(pato)lógico. No obstante, es igualmente cierto que, en un porcentaje reducido de jóvenes (aproximadamente un 10-30\%), estas experiencias pueden ser persistentes, causar malestar y reducir la funcionalidad y calidad de vida. Dichas experiencias predicen la aparición de un trastorno psicótico posterior (tasa $0.5 \%$ por año), particularmente si son persistentes (van Os y Linscott, 2012). No obstante, la comprensión sobre los predictores que favorecen la persistencia de estas experiencias en adolescentes es aún muy limitada (Kalman et al., 2019).

En segundo lugar, se tiene que examinar su impacto clínico, analizando la frecuencia y persistencia temporal y el grado de preocupación, intensidad y malestar asociado, así como si estas experiencias se asocian con otros signos o síntomas psicopatológicos (p.ej., ansiedad, depresión). Al mismo tiempo, es necesario recoger información sobre la búsqueda de ayuda y necesidad de tratamiento por parte de la persona (familia, escuela, etc.), su contexto familiar y analizar el posible impacto funcional o disminución significativa del funcionamiento escolar, social y ocupacional. En relación con lo anterior, las experiencias psicóticas de cierta gravedad, aunque no alcancen el nivel clínico, suelen aparecer también en diversos problemas psicopatológicos como la depresión o los trastornos de la personalidad. Las experiencias psicóticas per se tienen, por lo tanto, un carácter transdiagnóstico (van Os y Reininghaus, 2016). En este sentido, se debería evaluar este tipo de experiencias en otros problemas psicopatológicos más allá de la psicosis e incluso, por ejemplo, en cribados de salud mental en la población general.

En tercer lugar, se debe contemplar en la ecuación el rol de diversos factores como, por ejemplo, la historia previa de experiencias de trauma (p.ej., abuso sexual, bullying), consumo de sustancias (p.ej., cannabis), los estilos de afrontamiento y el estrés (Espina y Calvete, 2017) o la desregulación afectiva. En este sentido, se hace necesario analizar su relación e interacción con otras variables procedentes de múltiples niveles de análisis, tanto factores de riesgo como factores protectores de la persona. Igualmente, el juicio clínico es además 
de relevante, necesario. Por ello, se pueden utilizar otras entrevistas, escalas o procedimiento que permitan explorar de forma sistemática la topografía de estas experiencias. También se deberían considerar las posibles barreras y dificultades (p.ej., el estigma del entorno, el autoestigma, los problemas de introspección) que puede presentar la persona adolescente a la hora de responder a este tipo de autoinformes o, incluso, hablar de este tipo de experiencias. Asimismo, es importante establecer un diagnóstico diferencial con otros cuadros psicopatológicos (p.ej., trastornos del espectro autista), enfermedades neurológicas (p.ej., tumores o epilepsias), trastornos metabólicos, o psicosis tóxicas (por abuso de sustancias o debido a medicación). Como se ha comentado, las experiencias psicóticas (p.ej., alucinaciones) no se circunscriben únicamente a los trastornos del espectro psicótico. Dada esta complejidad, se recomienda que la evaluación y posterior intervención, si fuera necesaria, la lleve a cabo un psicólogo experto en esta temática.

En cuarto lugar, resulta preciso reconocer que el PQ-B es un cuestionario tipo autoinforme, con las consabidas inherentes limitaciones a este tipo de herramientas (p.ej., sesgos de respuesta). Además, recoge únicamente información de la dimensión positiva del fenotipo psicótico, esto es, no considera la dimensión negativa (p.ej., anhedonia) ni la de desorganización cognitiva (p.ej., pensamiento desorganizado). Asimismo, como cualquier instrumento de evaluación, no está libre de error de medida, lo cual se puede asociar a falsos positivos y negativos. También es necesario mencionar que los baremos presentados en el presente trabajo han sido extraídos de una única comunidad autónoma española (La Rioja), por lo que se debe tener cierta cautela a la hora de la extrapolación de los resultados a otros grupos de interés.

En suma, el PQ-B parece ser un instrumento de medida breve, sencillo, útil y con adecuadas propiedades psicométricas para la valoración de las experiencias psicóticas y/o el cribado del riesgo de psicosis en población adolescente española. Su reducido número de ítems y la valoración conjunta que ofrece, tanto de la frecuencia como del malestar asociado a las experiencias, lo convierten en un test interesante para usar como prueba de screening en la población general o en circunstancias donde escasean los recursos temporales y/o materiales, pero se desea obtener información de este fenómeno. Futuros trabajos deberían analizar las experiencias psicóticas en estudios micro longitudinales, de la mano de la evaluación ecológica momentánea, así como examinar su validez predictiva en muestras de participantes españoles.

\section{Conflicto de intereses}

Los autores declaran que no existen conflictos de intereses.

\section{Referencias}

Bak, M., Krabbendam, L., Janssen, I., de Graaf, R., Vollebergh, W., \& van Os, J. (2005). Early trauma may increase the risk for psychotic experiences by impacting on emotional response and perception of control. Acta Psychiatrica Scandinavica, 112, 360-366. https://doi.org/10.1111/j.1600-0447.2005.00646.x

Borsboom, D. (2017). A network theory of mental disorders. World Psychiatry, 16, 5-13.

Calkins, M. E., Moore, T. M., Merikangas, K. R., Burstein, M., Satterthwaite, T. D., Bilker, W. B., Kosha, R. Chiavacci, D. Wolf, D. H., Mentch, F., Qiu, H., Connolly, J. J., Sleiman, P.A., Hakonarson, H., Gur, R.C., \& Gur, R. E. (2014). The psychosis spectrum in a young U.S. community sample: Findings from the Philadelphia Neurodevelopmental Cohort. World Psychiatry, 13(3), 296-305. https://doi.org/10.1002/wps.20152

Cicero, D. C., Krieg, A., \& Martin, E. A. (2019). Measurement Invariance of the Prodromal Questionnaire-Brief Among White, Asian, Hispanic, and Multiracial Populations. Assessment, 26, 294-304. https://doi.org/10.1177/1073191116687391

Cooke, A. (2014). Comprender la Psicosis. (Edición española). The British Psychological Society (Divison of Clinical Psychology).

Cougnard, A., Marcelis, M., Myin-Germeys, I., De Graaf, R., Vollebergh, W., Krabbendam, L., \& Van Os, J. (2007). Does normal developmental expression of psychosis combine with environmental risk to cause persistence of psychosis? A psychosis proneness-persistence model. Psychological Medicine, 37, 513-527. https://.doi.org/10.1017/S0033291706009731

Debbané, M., Eliez, S., Badoud, D., Conus, P., Flückiger, R., \& Schultze-Lutter, F. (2015). Developing psychosis and its risk states through the lens of schizotypy. Schizophrenia Bulletin, 41, 396-407. https://.doi.org/10.1093/schbul/sbu176

Dolphin, L., Dooley, B., \& Fitzgerald, A. (2015). Prevalence and correlates of psychotic like experiences in a nationally representative community sample of adolescents in Ireland. Schizophrenia Research, 169, 217-241. https://.doi.org/10.1016/ j.schres.2015.09.005

Espina, M. y Calvete, E. (2017). Estilos de afrontamiento y generación de estrés interpersonal en adolescentes. Revista de Psicopatología y Psicología Clínica, 22, 21-32. https://doi. org/10.5944/rppc.vol.22.num.1.2017.16825

Fernández-León, S., Senín-Calderón, C., Gutiérrez-López, M. L., \& Rodríguez-Testal, J. F. (2019). Spanish validation of the Aberrant Salience Inventory in a general adolescent population. Psicothema, 3l(2), 210-217. https://doi.org/ 10.7334/psicothema2018.308

Fisher, H. L., Caspi, A., Poulton, R., Meier, M. H., Houts, R., Harrington, H., Arseneault, L \& Moffitt, T. E. (2013). Specificity of childhood psychotic symptoms for predicting schizophrenia by 38 years of age: a birth cohort study. Psychological Medicine, 43, 2077-2086. https://doi. org/10.1017/S0033291712003091 
Fonseca-Pedero, E., Ortuño-Sierra, J., Inchausti, F., RodríguezTestal, J. F., \& Debbané, M. (2020). Beyond clinical high-risk state for psychosis: the network structure of multidimensional psychosis liability in Adolescents. Frontiers of Psychiatry, 10.967. https://doi.org/10.3389/fpsyt.2019.00967

Fonseca-Pedrero, E. (2018a). Análisis de redes en psicología. Papeles del Psicologo, 39, 1-12.

Fonseca-Pedrero, E. (Ed.) (2018b). Evaluación de los trastornos del espectro psicótico. Pirámide.

Fonseca-Pedrero, E. (Ed.) (2019). Tratratamientos psicológicos para la psicosis. Pirámide.

Fonseca-Pedrero, E., Debbané, M., Ortuño-Sierra, J., Chan, R. C. K., Cicero, D. C., Zhang, L. C., ... Jablensky, A. (2018). The structure of schizotypal personality traits: A cross-national study. Psychological Medicine, 48, 451-462. https://doi. org/10.1017/S0033291717001829

Fonseca-Pedrero, E, Gooding, D. C., Ortuño-Sierra, J., \& Paino, M. (2016). Assessing self-reported clinical high risk symptoms in community-derived adolescents: A psychometric evaluation of the Prodromal Questionnaire-Brief. Comprehensive Psychiatry, 66, 1-8. https://doi.org/10.1016/j.comppsych.2016.01.013

Fonseca-Pedrero, E., Gooding, D., Debbané, M., \& Muñiz, J. (2016). Psychopathology: Psychosis assessment and high-risk paradigms. En F. T. L. Leong, D. Bartram, F. Cheung, K. F. Geisinger, \& D. Iliescu (Eds.), The ITC International Handbook of Testing and Assessment (pp. 147-170). Oxford University Press.

Fonseca-Pedrero, E, Santarén-Rosell, M., Lemos-Giráldez, S., Paino, M., Sierra-Baigrie, S., y Muñiz, J. (2011). Síntomas psicóticos subclínicos en población general adolescente. Actas Españolas de Psiquiatría, 39, 155-162.

Fonseca-Pedrero, E., \& Inchausti, F. (2018). An update in the prevention of psychosis spectrum disorders. Papeles del Psicologo, 39, 127-139. https://doi.org/10.23923/pap. psicol2018.2860

Fonseca-Pedrero, E, Inchausti, F., Pérez-Albéniz, A., \& OrtuñoSierra, J. (2018). Validation of the Prodromal QuestionnaireBrief in a representative sample of adolescents: Internal structure, norms, reliability, and links with psychopathology. International Journal of Methods in Psychiatric Research, 27, e1740. https://doi.org/10.1002/mpr.1740

Fonseca-Pedrero, E., Paino-Piñeiro, M., Lemos-Giráldez, S., Villazón-García, U., \& Muñiz, J. (2009). Validation of the Schizotypal Personality Questionnaire-Brief Form in adolescents. Schizophrenia Research, 111(1-3), 53-60. https://doi.org/10.1016/j.schres.2009.03.006

Fonseca-Pedrero, E., Debbané, M., Rodríguez-Testal, J. F., Cohen, A. S., Docherty, A. R., \& Ortuño-Sierra, J. (2021). Schizotypy: The Way Ahead. Psicothema, 33(1), 16-27. https://doi. org/10.7334/psicothema2019.285

Fusar-Poli, P, Carpenter, W. T., Woods, S. W., \& McGlashan, T. H. (2014). Attenuated Psychosis Syndrome: Ready for DSM5.1? Annual Review of Clinical Psychology, 10, 155-192. https://doi.org/10.1146/annurev-clinpsy-032813-153645

Fusar-Poli, P, Tantardini, M., De Simone, S., Ramella-Cravaro, V., Oliver, D., Kingdon, J., Kotlicka-Antczak, M., Valmaggia, L., Lee, J., Millan, M. J., Galderisi, S., Balottin, U., Ricca, V., \& McGuire, P. (2017). Deconstructing vulnerability for psychosis: Meta-analysis of environmental risk factors for psychosis in subjects at ultra high-risk. European Psychiatry, 40, 65-75. https://doi.org/10.1016/j.eurpsy.2016.09.003

Fusar-Poli, P., McGorry, P. D., \& Kane, J. M. (2017). Improving outcomes of first-episode psychosis: an overview. World Psychiatry, 16(3), 251-265. https://doi.org/10.1002/wps.20446

Fusar-Poli, P., Salazar de Pablo, G., Correll, C. U., MeyerLindenberg, A., Millan, M. J., Borgwardt, S., Galderisi, S., Bechdolf, A., Pfennig, A., Kessing, L. V., van Amelsvoort , T., Nieman, D. H., Domschke, K., Krebs, M. O., Koutsouleris, N., McGuire, P., Do, K.Q, \& Arango, C. (2020). Prevention of Psychosis: Advances in Detection, Prognosis, and Intervention. JAMA Psychiatry, 77, 755-765. https://doi.org/10.1001/ jamapsychiatry.2019.4779

Hernández, A., Ponsoda, V., Muñiz, J., Prieto, G., y Elosua, P. (2016). Revisión del modelo para evaluar la calidad de los tests utilizados en España. Papeles del Psicólogo, 37, 192197. http://dx.doi.org/10.1027/1015-5759.12.1.62

Howes, O. D., \& Murray, R. M. (2014). Schizophrenia: An integrated sociodevelopmental-cognitive model. Lancet, 383(9929), 1677-1687. https://doi.org/10.1016/S01406736(13)62036-X

Kahn, R. S., Sommer, I. E., Murray, R. M., Meyer-Lindenberg, A., Weinberger, D. R., Cannon, T. D., O’Donovan, M., Correll, C.U., Kane, J. M., van Os, J., \& Insel, T. R. (2015). Schizophrenia. Nature Reviews Disease Primers, 1, 15067. https://doi.org/10.1038/nrdp.2015.67

Kalman, J. L., Bresnahan, M., Schulze, T. G., \& Susser, E. (2019). Predictors of persisting psychotic like experiences in children and adolescents: A scoping review. Schizophrenia research, 209, 32-39. https://doi.org/10.1016/j.schres.2019.05.012

Kaymaz, N., Drukker, M., Lieb, R., Wittchen, H. U., Werbeloff, N., Weiser, M., \& van Os, J. (2012). Do subthreshold psychotic experiences predict clinical outcomes in unselected non-helpseeking population-based samples? A systematic review and meta-analysis, enriched with new results. Psychological Medicine, 20, 1-15. https://doi.org/10.1017/S0033291711002911

Kelleher, I., Connor, D., Clarke, M. C., Devlin, N., Harley, M., \& Cannon, M. (2012). Prevalence of psychotic symptoms in childhood and adolescence: a systematic review and metaanalysis of population-based studies. Psychological Medicine, 9, 1-7. https://doi.org/10.1017/S0033291711002960

Kelleher, I., Keeley, H., Corcoran, P., Ramsay, H., Wasserman, C., Carli, V., Sarchiapone, M., Hoven, C., Wasserman, D., \& Cannon, M. (2013). Childhood Trauma and Psychosis in a Prospective Cohort Study: Cause, Effect, and Directionality. American Journal of Psychiatry, 170, 734-741. https://doi. org/10.1176/appi.ajp.2012.12091169

Kline, E., \& Schiffman, J. (2014). Psychosis risk screening: A systematic review. Schizophrenia Research, 158, 11-18. https://doi.org/10.1016/j.schres.2014.06.036

Kwapil, T. R., \& Barrantes-Vidal, N. (2015). Schizotypy: Looking back and moving forward. Schizophrenia Bulletin, 41, S366373. https://doi.org/10.1093/schbul/sbu186

Lemos Giráldez, S., Fonseca-Pedrero, E., Paino, M., y Vallina, O. (2015). Esquizofrenia y otros trastornos psicóticos. Síntesis.

Linscott, R. J., \& van Os, J. (2013). An updated and conservative systematic review and meta-analysis of epidemiological evidence on psychotic experiences in children and adults: on the pathway from proneness to persistence to dimensional 
expression across mental disorders. Psychological Medicine, 43, 1133-1149. https://doi.org/10.1017/s0033291712001626

Loewy, R. L., Bearden, C. E., Johnson, J. K., Raine, A., \& Cannon, T. D. (2005). The Prodromal Questionnaire (PQ): preliminary validation of a self-report screening measure for prodromal and psychotic syndromes. Schizophrenia Research, 79, 117125. https://doi.org/10.1016/j.schres.2005.03.007

Loewy, R. L., Pearson, R., Vinogradov, S., Bearden, C. E., \& Cannon, T. D. (2011). Psychosis risk screening with the Prodromal Questionnaire--brief version (PQ-B). Schizophrenia Research, 129, 42-46. https://doi.org/10.1016/j.schres.2011.03.029

McGrath, J. J., Saha, S., Al-Hamzawi, A., Alonso, J., Bromet, E. J., Bruffaerts, R., Caldas-de-Almeida, J.M., Chiu, W. T., de Jonge, P., Fayyad, J., Florescu, S., Gureje, O., Haro, J. M., Hu, C., Kovess-Masfety, V., Lepine, J. P., Lim, C.C.W., Medina Mora, M. E., ...Kessler, R. C. (2015). Psychotic experiences in the general population: a cross-national analysis based on 31.261 respondents from 18 countries. JAMA Psychiatry, 72, 697-705. https://doi.org/10.1001/jamapsychiatry.2015.0575

Millan, M. J., Andrieux, A., Bartzokis, G., Cadenhead, K., Dazzan, P., Fusar-Poli, P., Gallinat, J., Giedd, J., Grayson, D. R., Heinrichs, M., Kahn, R., Krebs, M. O., Leboyer, M., Lewis, D., Marin, O., Marin, P., Meyer-Lindenberg, A., McGorry, P., McGuire, P., ...Weinberger, D. (2016). Altering the course of schizophrenia: progress and perspectives. Nature Reviews Drug Discovery, 15(7), 485-515. https://doi.org/10.1038/ nrd.2016.28

Muñiz, J., y Fonseca-Pedrero, E. (2019). Diez pasos para la construcción de un test. Psicothema, 31, 7-16. http://dx.doi. org/10.7334/psicothema2018.291

Muñiz, J., Hernández, A., y Fernández-Hermida, J. R. (2020). Utilización de los test en España: el punto de vista de los psicólogos. Papeles del Psicólogo, 41, 1-15. https://doi. org/10.23923/pap.psicol2020.2921

Nuevo, R., Chatterji, S., Verdes, E., Naidoo, N., Arango, C., \& Ayuso-Mateos, J. L. (2012). The continuum of psychotic symptoms in the general population: A cross-national study. Schizophrenia Bulletin, 38, 475-485 https://doi.org/10.1093/ schbul/sbq099

Ord, L., Myles-Worsley, M., Blailes, F., \& Ngiralmau, H. (2004). Screening for prodromal adolescents in an isolated high-risk population. Schizophrenia Research, 71, 507-508. https://doi. org/10.1016/j.schres.2004.03.014

Pérez-Álvarez, M. (2018). Para pensar la psicología más allá de la mente y el cerebro: Un enfoque transteórico. Papeles del
Psicólogo, 39, 161-173. https://doi.org/10.23923/pap.psicol2018. 2875

Pérez-Álvarez, M. (2012). Las raíces de la psicopatología moderna: La melancolía y la esquizofrenia. Pirámide.

Pérez-Álvarez, M., y García-Montes, J. M. (2018). Evaluacion fenomenológica más allá de los síntomas. In Evaluación de los trastornos del espectro psicótico (pp. 331-363). Pirámide.

Pére-Álvarez, M., Montes-García, J. M., y Louis, S. (2010). La hora de la fenomenología en la esquizofrenia. Clínica y Salud, 21(3), 221-233. https://doi.org/10.5093/c12010v21n3a2

Radua, J., Ramella-Cravaro, V., Ioannidis, J. P. A., Reichenberg, A., Phiphopthatsanee, N., Amir, T., Thoo, H. Y., Oliver, D., Davies, C., Morgan, C., McGuire, P., Murray, R. M., \& FusarPoli, P. (2018). What causes psychosis? An umbrella review of risk and protective factors. World Psychiatry, 17, 49-66. https://doi.org/10.1002/wps.20490

Savill, M., D’Ambrosio, J., Cannon, T. D., \& Loewy, R. L. (2018). Psychosis risk screening in different populations using the Prodromal Questionnaire: A systematic review. Early Intervention in Psychiatry, 12, 3-14. https://doi.org/10.1111/ eip. 12446

Stefanis, N. C., Hanssen, M., Smirnis, N. K., Avramopoulos, D. A., Evdokimidis, I. K., Stefanis, C. N., Verdoux, H., \& van Os, J. (2002). Evidence that three dimensions of psychosis have a distribution in the general population. Psychological Medicine, 32(2), 347-358. https://doi.org/10.1017/S0033291701005141

Strauss, G. P., Raugh, I. M., Mittal, V. A., Gibb, B. E., \& Coles, M. E. (2018). Bullying victimization and perpetration in a community sample of youth with psychotic like experiences. Schizophrenia Research, 195, 534-536. https://doi. org/10.1016/j.schres.2017.08.056

van Os, J., \& Linscott, R. J. (2012). Introduction: The extended psychosis phenotype - Relationship with schizophrenia and with ultrahigh risk status for psychosis. Schizophrenia Bulletin, 38(2), 227-230. https://doi.org/10.1093/schbul/sbr188

van Os, J., \& Reininghaus, U. (2016). Psychosis as a transdiagnostic and extended phenotype in the general population. World Psychiatry, 15(2), 118-124. https://doi.org/10.1002/wps.20310

Zammit, S., Kounali, D., Cannon, M., David, A. S., Gunnell, D., Heron, J., Jones, P., Lewis, S., Sullivan, S., Wolke, D., \& Lewis, G. (2013). Psychotic experiences and psychotic disorders at age 18 in relation to psychotic experiences at age 12 in a longitudinal population-based cohort study. American Journal of Psychiatry, 170, 742-750. https://doi.org/10.1176/ appi.ajp.2013.12060768 


\section{Anexo 1. Versión española del Prodromal Questionnaire-Brief}

Por favor, indique si ha tenido los siguientes pensamientos. sensaciones y experiencias durante el pasado mes marcando «Sí» o «No» en cada pregunta. No incluya experiencias que tienen lugar bajo la influencia del alcohol, las drogas o los medicamentos que le hayan sido prescritos. Si contesta «Si» a una pregunta, indique también qué malestar le ha producido dicha experiencia.

1. ¿Los ambientes conocidos le parecen a veces extraños, confusos, amenazantes o irreales?

$$
\square \text { SI } \square \text { NO }
$$

Si contestó Sí: Cuando esto sucede, me siento asustado. preocupado o me causa problemas:

$\square$ Totalmente en desacuerdo $\square$ en desacuerdo $\square$ neutral $\square$ de acuerdo $\square$ totalmente de acuerdo

2. ¿Alguna vez ha percibido sonidos inusuales como estallidos, chasquidos, silbidos, aplausos o timbres en sus oídos? $\square$ SI $\square$ NO

Si contestó Sí: Cuando esto sucede, me siento asustado. preocupado o me causa problemas:

$\square$ Totalmente en desacuerdo $\square$ en desacuerdo $\square$ neutral $\square$ de acuerdo $\square$ totalmente de acuerdo

3. ¿Las cosas que ve le parecen diferentes a como normalmente son (más brillantes o más apagadas, más grandes o más pequeñas, o con cambios en algún otro aspecto)?

$$
\square \text { SI } \square \text { NO }
$$

Si contestó Sí: Cuando esto sucede, me siento asustado. preocupado o me causa problemas:

$\square$ Totalmente en desacuerdo $\square$ en desacuerdo $\quad \square$ neutral $\quad \square$ de acuerdo $\quad \square$ totalmente de acuerdo

4. ¿Ha tenido experiencias de telepatía, poderes de vidente o de adivino?

$$
\square \text { SI } \square \text { NO }
$$

Si contestó Sí: Cuando esto sucede, me siento asustado. preocupado o me causa problemas:

$\square$ Totalmente en desacuerdo $\square$ en desacuerdo $\square$ neutral $\square$ de acuerdo $\square$ totalmente de acuerdo

5. ¿Alguna vez ha sentido como si no tuviera control de sus propias ideas o pensamientos?

$$
\square \text { SI } \square \text { NO }
$$

Si contestó Sí: Cuando esto sucede, me siento asustado. preocupado o me causa problemas:

$\square$ Totalmente en desacuerdo $\square$ en desacuerdo $\square$ neutral $\square$ de acuerdo $\square$ totalmente de acuerdo

6. ¿Tiene dificultad para seguir su propio tema, debido a que divaga o pierde mucho la pista cuando habla?

$$
\square \text { SI } \square \text { NO }
$$

Si contestó Sí: Cuando esto sucede, me siento asustado. preocupado o me causa problemas:

$\square$ Totalmente en desacuerdo $\square$ en desacuerdo $\square$ neutral $\square$ de acuerdo $\square$ totalmente de acuerdo

7. ¿Tiene la fuerte sensación o la creencia de que posee alguna clase de dones o talentos inusuales?

$$
\square \text { SI } \square \text { NO }
$$

Si contestó Sí: Cuando esto sucede, me siento asustado preocupado o me causa problemas:

$\square$ Totalmente en desacuerdo $\square$ en desacuerdo $\square$ neutral $\square$ de acuerdo $\square$ totalmente de acuerdo

8. ¿Tiene la sensación de que otras personas le observan o hablan de usted?

$$
\square \text { SI } \square \text { NO }
$$

Si contestó Sí: Cuando esto sucede, me siento asustado preocupado o me causa problemas:

$\square$ Totalmente en desacuerdo $\square$ en desacuerdo $\square$ neutral $\square$ de acuerdo $\square$ totalmente de acuerdo

9. ¿Nota a veces extrañas sensaciones en la piel o debajo de ella, como bichos reptando?

$$
\square \text { SI } \square \text { NO }
$$

Si contestó Sí: Cuando esto sucede, me siento asustado. preocupado o me causa problemas:

$\square$ Totalmente en desacuerdo $\square$ en desacuerdo $\quad \square$ neutral $\quad \square$ de acuerdo $\square$ totalmente de acuerdo

10. ¿Se siente a veces repentinamente distraído por sonidos distantes de los que normalmente no se da cuenta? $\square$ SI $\square$ NO

Si contestó Sí: Cuando esto sucede, me siento asustado. preocupado o me causa problemas:

$\square$ Totalmente en desacuerdo $\square$ en desacuerdo $\quad \square$ neutral $\quad \square$ de acuerdo $\quad \square$ totalmente de acuerdo

11. ¿Alguna vez ha tenido la sensación de que había alguna persona o fuerza a su alrededor, aunque no podía ver a nadie? $\square$ SI $\square$ NO

Si contestó Sí: Cuando esto sucede, me siento asustado. preocupado o me causa problemas:

$\square$ Totalmente en desacuerdo $\square$ en desacuerdo $\quad \square$ neutral $\quad \square$ de acuerdo $\square$ totalmente de acuerdo 
12. ¿Le preocupa a veces que algo pueda ir mal en su mente? $\square$ SI $\square$ NO

Si contestó Sí: Cuando esto sucede, me siento asustado. preocupado o me causa problemas:

$\square$ Totalmente en desacuerdo $\square$ en desacuerdo $\square$ neutral $\square$ de acuerdo $\square$ totalmente de acuerdo

13. ¿Ha sentido alguna vez que no existía, que el mundo no existía o que estaba muerto? $\square$ SI $\square$ NO

Si contestó Sí: Cuando esto sucede, me siento asustado preocupado o me causa problemas:

$\square$ Totalmente en desacuerdo $\square$ en desacuerdo $\quad \square$ neutral $\quad \square$ de acuerdo $\square$ totalmente de acuerdo

14. ¿Se ha sentido a veces confuso sobre si algo de lo que le pasaba era real o imaginario? $\square$ SI $\square$ NO

Si contestó Sí: Cuando esto sucede, me siento asustado preocupado o me causa problemas:

$\square$ Totalmente en desacuerdo $\square$ en desacuerdo $\quad \square$ neutral $\quad \square$ de acuerdo $\square$ totalmente de acuerdo

15. ¿Tiene creencias que a otras personas les parecerían extrañas o inusuales? $\square$ SI $\square$ NO

Si contestó Sí: Cuando esto sucede, me siento asustado. preocupado o me causa problemas:

$\square$ Totalmente en desacuerdo $\square$ en desacuerdo $\quad \square$ neutral $\quad \square$ de acuerdo $\quad \square$ totalmente de acuerdo

16. ¿Siente que partes de su cuerpo han cambiado de alguna manera o que partes de su cuerpo están funcionando de manera diferente?

$\square$ SI $\square$ NO

Si contestó Sí: Cuando esto sucede, me siento asustado preocupado o me causa problemas:

$\square$ Totalmente en desacuerdo $\square$ en desacuerdo $\square$ neutral $\quad \square$ de acuerdo $\square$ totalmente de acuerdo

17. ¿Sus pensamientos son a veces tan intensos que casi puede oírlos? $\square$ SI $\quad \square$ NO

Si contestó Sí: Cuando esto sucede, me siento asustado. preocupado o me causa problemas:

$\square$ Totalmente en desacuerdo $\square$ en desacuerdo $\quad \square$ neutral $\quad \square$ de acuerdo $\square$ totalmente de acuerdo

18. ¿Experimenta sentimientos de recelo y desconfianza hacia otras personas? $\square$ SI $\square$ NO

Si contestó Sí: Cuando esto sucede, me siento asustado. preocupado o me causa problemas:

$\square$ Totalmente en desacuerdo $\square$ en desacuerdo $\quad \square$ neutral $\quad \square$ de acuerdo $\square$ totalmente de acuerdo

19. ¿Alguna vez ha visto cosas inusuales como flashes, llamas, luces deslumbrantes o figuras geométricas? $\square$ SI $\square$ NO

Si contestó Sí: Cuando esto sucede, me siento asustado. preocupado o me causa problemas:

$\square$ Totalmente en desacuerdo $\square$ en desacuerdo $\quad \square$ neutral $\quad \square$ de acuerdo $\square$ totalmente de acuerdo

20. ¿Alguna vez ha visto cosas que otras personas no pueden ver o no parecen ver? $\square$ SI $\square$ NO

Si contestó Sí: Cuando esto sucede, me siento asustado. preocupado o me causa problemas:

$\square$ Totalmente en desacuerdo $\square$ en desacuerdo $\quad \square$ neutral $\quad \square$ de acuerdo $\square$ totalmente de acuerdo

21. ¿A veces a la gente le cuesta entender lo que está diciendo? $\square$ SI $\square$ NO

Si contestó Sí: Cuando esto sucede, me siento asustado preocupado o me causa problemas:

$\square$ Totalmente en desacuerdo $\quad \square$ en desacuerdo $\quad \square$ neutral $\quad \square$ de acuerdo $\quad \square$ totalmente de acuerdo

Forma de corrección:

Se pueden calcular dos puntuaciones totales, por un lado, la puntuación total de frecuencia (opción sí/no) y, por otro, la puntuación total de malestar (opciones desde totalmente en desacuerdo a totalmente de acuerdo):

a) Puntuación total de frecuencia: sumatorio de las puntuaciones en los 21 ítems con la codificación $\mathrm{Sí}=1$ y No=0.

b) Puntuación total de distrés: sumatorio de las puntuaciones en los 21 ítems siguiendo la codificación: Totalmente en desacuerdo $=0$; en desacuerdo $=1$; neutral $=2$; de acuerdo $=3$; totalmente de acuerdo $=4$. 